\title{
SEX-RATIO CONFLICT BETWEEN QUEENS AND WORKERS IN EUSOCIAL HYMENOPTERA: MECHANISMS, COSTS, AND THE EVOLUTION OF SPLIT COLONY SEX RATIOS
}

\author{
Ken R. Helms, ${ }^{1,2,3}$ Max Reuter, ${ }^{1,4}$ and Laurent Keller ${ }^{1}$ \\ ${ }^{1}$ Department of Ecology and Evolution, University of Lausanne, 1015 Lausanne, Switzerland \\ ${ }^{2}$ Department of Biology, University of Vermont, Burlington, Vermont 05405
}

\begin{abstract}
Because workers in the eusocial Hymenoptera are more closely related to sisters than to brothers, theory predicts that natural selection should act on them to bias (change) sex allocation to favor reproductive females over males. However, selection should also act on queens to prevent worker bias. We use a simulation approach to analyze the coevolution of this conflict in colonies with single, once-mated queens. We assume that queens bias the primary (egg) sex ratio and workers bias the secondary (adult) sex ratio, both at some cost to colony productivity. Workers can bias either by eliminating males or by directly increasing female caste determination. Although variation among colonies in kin structure is absent, simulations often result in bimodal (split) colony sex ratios. This occurs because of the evolution of two alternative queen or two alternative worker biasing strategies, one that biases strongly and another that does not bias at all. Alternative strategies evolve because the mechanisms of biasing result in accelerating benefits per unit cost with increasing bias, resulting in greater fitness for strategies that bias more and bias less than the population equilibrium. Strategies biasing more gain from increased biasing efficiency whereas strategies biasing less gain from decreased biasing cost. Our study predicts that whether queens or workers evolve alternative strategies depends upon the mechanisms that workers use to bias the sex ratio, the relative cost of queen and worker biasing, and the rates at which queen and worker strategies evolve. Our study also predicts that population and colony level sex allocation, as well as colony productivity, will differ diagnostically according to whether queens or workers evolve alternative biasing strategies and according to what mechanism workers use to bias sex allocation.
\end{abstract}

Key words._Parent-offspring conflict, queen-worker conflict, sex allocation, split sex-ratio, theory.

Received March 22, 2005. Accepted September 29, 2005.

Studies of sex allocation in eusocial Hymenoptera (ants, bees, wasps) have resulted in important tests of evolutionary theories of sex allocation, inclusive fitness, and parent-offspring conflict. This importance arises from haplodiploid sex determination, which results in an asymmetry in relatedness in which workers are more closely related to sisters than to brothers (Hamilton 1964; Trivers and Hare 1976). As a consequence, workers favor a more female-biased sex allocation than the queen, leading to intracolony conflict over the sex ratio. Because workers are generally not mated and have limited opportunities for reproduction, their fitness results primarily from rearing eggs produced by their mother (the queen). As a result, indirect selection should act on workers to change, or bias, colony investment in favor of reproductive females. However, because queens are equally related to daughters and sons, selection should act on queens to prevent worker bias (Trivers and Hare 1976; Bourke and Franks 1995; Crozier and Pamilo 1996). Empirical studies have provided substantial evidence consistent with workers biasing investment and queens acting to prevent such bias, supporting the occurrence of this conflict (Trivers and Hare 1976; Nonacs 1986; Boomsma 1989; Bourke and Franks 1995; Crozier and Pamilo 1996; Chapuisat and Keller 1999; Beekman and Ratnieks 2003; Mehdiabadi et al. 2003).

Although asymmetrical relatedness of workers to females and males may explain global patterns of sex allocation

\footnotetext{
${ }^{3}$ Present address: Department of Biology, Marsh Life Sciences, University of Vermont, Burlington, Vermont 05405; E-mail: khelms@uvm.edu.

${ }^{4}$ Present address: Department of Biology, University College London, The Galton Laboratory, 4 Stephenson Way, London NW1 2HE, United Kingdom.
}

across populations, eusocial Hymenoptera exhibit another sex allocation characteristic of great significance: colonies within populations often produce highly divergent sex ratios. Some produce primarily or exclusively males and others produce primarily or exclusively females (Nonacs 1986; Boomsma and Grafen 1990). Theory has proposed that these bimodally distributed, or split, colony sex ratios result from facultative worker biasing in response to differences in relatedness asymmetry among colonies, and a number of studies support this hypothesis (e.g., Boomsma and Grafen 1990, 1991; Mueller 1991; Queller et al. 1993; Chan and Bourke 1994; Sundström 1994; Sundström et al. 1996; Queller and Strassmann 1998). Differences among colonies in relatedness asymmetry can arise from variation in queen mating frequency, partial worker reproduction, and variable numbers of reproductive queens per colony (e.g., Boomsma and Grafen 1990). However, split sex ratios are common even in populations of eusocial Hymenoptera where differences in relatedness asymmetry do not appear important (e.g., Hasegawa 1994; Pamilo and Seppä 1994; Vargo 1996; Helms 1999; Bourke and Ratnieks 2001; Passera et al. 2001; Fournier et al. 2003; Helms et al. 2004). In some cases, there is evidence that split sex ratios may result from queens exhibiting alternative biasing strategies, with some queens producing mostly male eggs and others mostly female eggs (e.g., Helms 1999; Helms et al. 2000; Bourke and Ratnieks 2001; Passera et al. 2001). We use the term alternative biasing strategies to refer to cases in which queens (or workers) exhibit two distinct pure strategies; for example, whereby some queens produce only male eggs whereas others produce both sexes at equal frequency (or in which some workers eliminate all males while others eliminate none). 
How polymorphisms in sex allocation can evolve in the absence of differences in relatedness asymmetry is largely unexplored. However, in a recent model, we explored the effect of costs of sex-ratio manipulation by workers on colony sex allocation (Reuter et al. 2004). We found that evolution leads to the emergence of two alternative worker strategies when the costs of biasing increase in a decelerating manner with increasing bias. One of these strategies is to manipulate the sex ratio up to complete female production and the other is to not manipulate at all. While providing an explanation for split sex ratios in the absence of relatedness asymmetry, that model assumed a constant primary sex ratio and did not allow for the coevolution of queen and workers strategies. Here we present a study that extends the previous analyses in several important ways. First, the present analysis allows for worker and queen strategies to coevolve in sex-ratio conflict, whereby the manipulation of investment by both parties is assumed to have a cost in terms of reduced colony productivity. Second, we explicitly model the two most likely mechanisms of worker sex ratio manipulation, that is the elimination of male brood versus the reallocation of workerdestined female larvae to the queen developmental pathway. Third, we address how the resolution of sex-ratio conflict is affected by the evolutionary trajectory that queen and worker strategies take during the process of coevolution. To do so, we use a simulation approach rather than analytical modeling. This approach allows us to manipulate the rate at which queen and worker biasing phenotypes evolve by varying the effect that mutant strategies have on queen and worker biasing. It also allows for analysis of the coevolutionary process. Our study provides a new approach to the study of sex allocation in eusocial Hymenoptera by investigating the importance of mechanisms and costs of sex-ratio biasing, and provides fundamental insights into the evolutionary mechanisms that can result in split colony sex ratios.

\section{Models of QueEn-Worker CONFlict}

We address the problem of coevolving queen and worker strategies using a simulation approach. During simulations, queen and worker strategies compete against each other over many generations, leading to stable coevolutionary equilibria. Our models assume that workers are sterile and all colonies have a single queen (monogyny) inseminated by a single male (monandry). We model the degree that queens and workers act to change (bias) the sex ratio as pure (nonfacultative) strategies and assume that they are effective only during the period(s) when reproductive offspring are produced. Queen and worker strategies are associated randomly according to their frequencies in the population. This implies that queen and worker strategies are genetically unlinked and completely independent in their expression; for example, a queen mechanism affecting the primary sex ratio (egg insemination rate) is presumed to have no phenotypic effect in sterile workers, whereas a worker mechanism affecting the secondary sex ratio (reproductive brood rearing) is presumed to have no phenotypic effect in queens (e.g., Lynch 1987; Mock and Parker 1997; Kölliker et al. 2000; Cheverud 2003). Colonies produce male and female sexuals (hereafter referred to simply as males and females) in proportions determined by the strat- egies applied by each colony's queen and workers. For simplicity, we assume that all colonies have equal lifetime resources and that the cost of producing a female is identical to the cost of producing a male. The assumption of equal production costs simplifies calculations by equating the numerical sex ratio with the investment sex ratio and has no effect on the evolutionary outcomes we address because selection acts on total investment in the sexes rather than their numbers, regardless of the cost of producing a male versus a female (e.g., Fisher 1958).

\section{Fitness of Queen and Worker Strategies}

The fitness of queen and worker strategies over one generation is modeled as a function of five parameters: the sex allocation in the colony (expressed as the proportion of a colony's resources invested in females, $f_{i}$ ), the sex allocation in the overall population $(F)$, the relatedness values to the sexuals produced (females: $r_{f}$, males: $r_{m}$ ), and the costs of sex-ratio biasing for queens and workers $\left(\gamma_{Q W}\right)$. All parameters are summarized in Table 1 . Using a standard notation (e.g., Boomsma and Grafen 1991; Pamilo 1991) we define fitness $(\alpha)$ of queens $\left(\alpha_{Q}\right)$ and workers $\left(\alpha_{W}\right)$ in colony $i$ within a population of colonies $(1 \ldots N)$ as:

$$
\alpha_{i}=r_{f} \cdot \frac{\left(1-\gamma_{Q W_{i}}\right) f_{i}}{\sum_{j=1}^{N}\left[\left(1-\gamma_{Q W_{j}}\right) f_{j}\right]}+r_{m} \frac{\left(1-\gamma_{Q W_{i}}\right)\left(1-f_{i}\right)}{\sum_{j=1}^{N}\left[\left(1-\gamma_{Q W_{j}}\right)\left(1-f_{j}\right)\right]} .
$$

In our models, we employ life-for-life values of relatedness (e.g., Grafen 1986), where relatedness of queens to females and males is 0.5 , whereas the relatedness of workers to females is 0.75 , and the relatedness of workers to males is 0.25 . These values imply outbreeding and a very large population size, which is realized in our simulations (see below).

\section{Biasing Mechanisms}

The effects of queen and worker biasing on colony sex allocation depend upon the mechanisms by which they bias. This section details the biasing mechanisms included in our simulations.

\section{Queens produce the primary sex ratio}

Our model assumes that queens alter colony sex allocation by varying the primary sex ratio (the sex ratio of eggs laid by the queen). The primary sex ratio has been proposed as a powerful way for queens to bias sex allocation (Bulmer 1981; Passera et al. 2001; Reuter and Keller 2001), and empirical evidence suggests that in a number of species queens do alter colony sex allocation by varying the primary sex ratio of male (haploid) and female (diploid) eggs (e.g., Aron et al. 1994, 1995; Keller et al. 1996; Passera et al. 2001). Queen variance in the temporal availability of haploid and diploid eggs (Pamilo and Rosengren 1983; Bourke and Ratnieks 2001) is equivalent to varying the primary sex ratio in our models.

We define the degree of queen sex-ratio bias, $B_{Q}$, as the deviation of the primary sex ratio from a value lambda, which 
TABLE 1. Summary of model parameters.

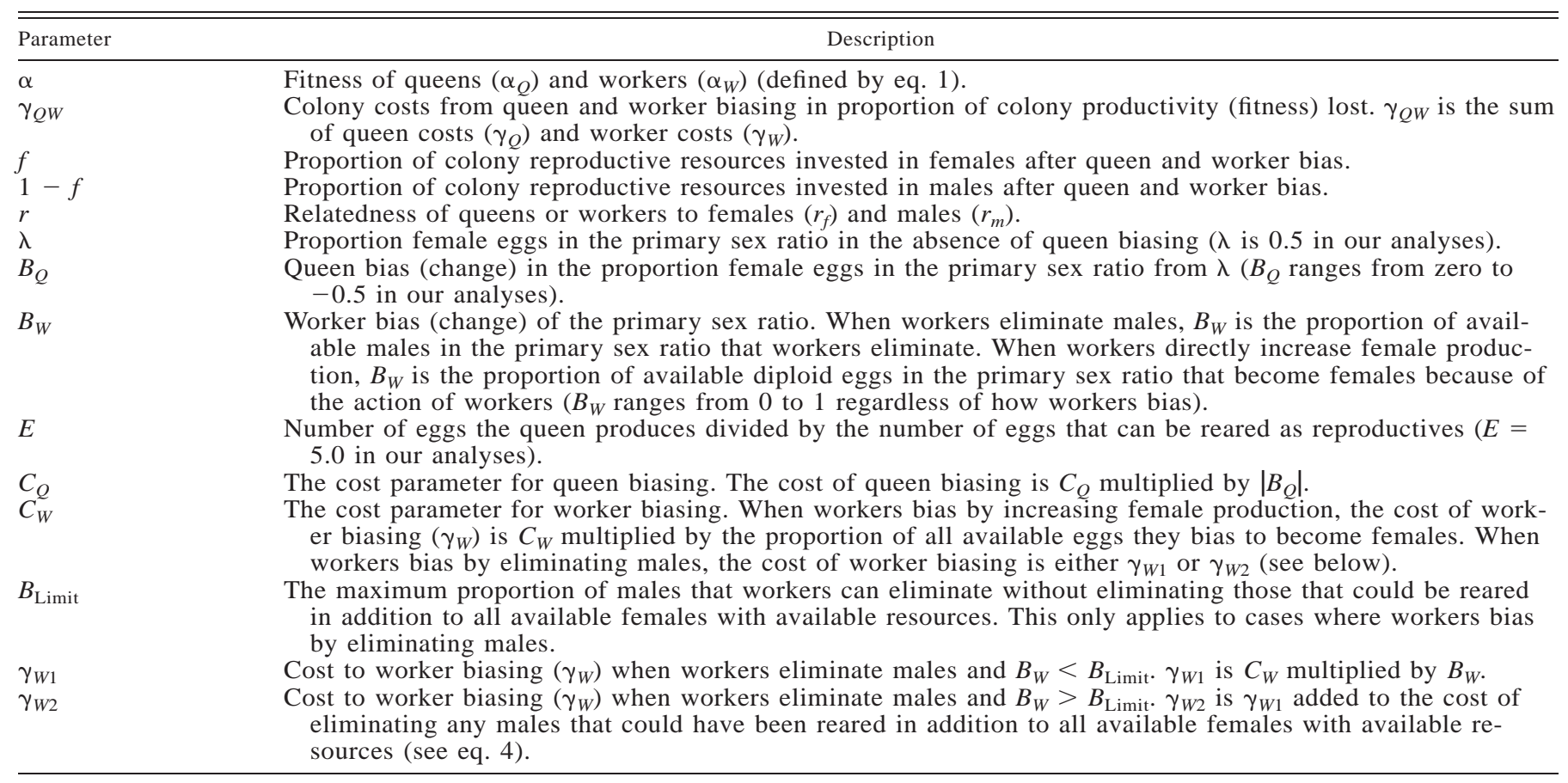

is the primary sex ratio in the absence of queen bias. The primary sex ratio (proportion females) produced by the queen in colony $i$ is then: $\lambda+B_{Q}$.

We only consider selection on queens to decrease the proportion of female brood and accordingly $B_{Q}$ can range from $-\lambda$ (only male eggs are produced) to zero (queens do not bias). This restriction in the parameter range is justified be-

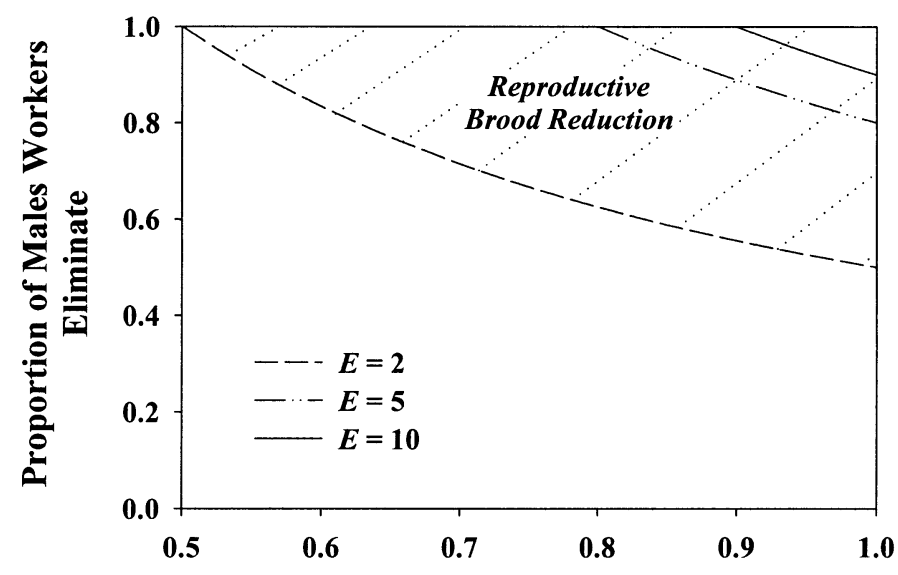

Proportion Male Eggs That Queen Produces

FIG. 1. The number of eggs that can be reared relative to the number the queen produces determines how much queens and workers can bias the sex ratio before they reduce the size of the reproductive brood. The primary sex ratio in the absence of queen biasing is assumed equal, and queen biasing ranges from no bias $(0.5)$ to producing all male eggs (1.0). The lines indicate where the female eggs available to rear are equal to those that can be reared (i.e., where $B_{W}=B_{\text {Limit }}$ (eq. 4) under three egg availabilities. $E$ is the number of eggs produced divided by the number of eggs potentially reared as reproductives. cause we assume the absence of local mate competition, a condition under which selection might act on queens to produce female-biased sex ratios (e.g., Hamilton 1967; Cremer and Heinze 2002).

We assume the default sex ratio lambda to be equal to one half. This value is the queen equilibrium sex ratio, and may be predicted prior to the evolution of queen-worker conflict (e.g., Fisher 1958). In addition, if queens exert no control over the primary sex ratio, a random mix of diploid and haploid eggs should, on average, occur, also resulting in an equal frequency of male and female eggs. Varying lambda in analyses showed that values differing from one half did not result in qualitatively different conclusions from those we present.

Queen egg production.-A fundamental, yet rarely addressed factor in queen-worker conflict is the availability of eggs (Pamilo 1991; Reuter and Keller 2001; Roisin and Aron 2003). In fact, workers cannot adaptively bias sex allocation unless eggs produced by the queen are in excess of those that can be reared as reproductives with available resources. Otherwise, worker bias of the sex ratio would only reduce reproductive output. To describe the availability of surplus eggs in the colony we define a parameter $E$ as the ratio of the number of eggs produced by the queen over the number that can be reared as reproductives. For the reason outlined above, $E$ must be greater than 1.0 for the evolution of worker biasing to evolve by natural selection. In our analyses, we assume that $E=5$ and eggs are fairly abundant relative to those that can be reared as reproductives. For simplicity, we also assume that $E$ does not vary among colonies. How queen and worker biasing affects brood reduction under different values of $E$ is illustrated in Figure 1. 


\section{Worker biasing mechanisms}

Workers can alter the primary sex ratio produced by the queen before sexuals are produced to result in a secondary, or final reproductive sex ratio. Two general mechanisms seem most likely: workers can eliminate males and redirect resources to the production of females or they can influence the caste determination of diploid eggs to increase the production of females (Pamilo 1991; Passera and Aron 1996; Reuter and Keller 2001; Hammond et al. 2002). We will determine the effects of these different mechanisms with separate analyses.

When workers eliminate males.-We assume that workers eliminate some proportion of males in the primary sex ratio and then rear the remaining males (along with females) at the proportion in which they occur in the remaining brood. So long as diploid eggs occur, increasing male elimination indirectly results in increasing the proportion of females in the reproductive brood.

If $B_{W}$ is the proportion of males in the primary sex ratio that workers eliminate, the secondary sex ratio following worker bias in colony $i$ ( $f_{i}$; eq. 1$)$ is given by

$$
f_{i}=\frac{\left(\lambda+B_{Q_{i}}\right)}{\left(\lambda+B_{Q_{i}}\right)+\left[1-\left(\lambda+B_{Q_{i}}\right)\right]-B_{W_{i}}\left[1-\left(\lambda+B_{Q_{i}}\right)\right]}
$$

where $\lambda+B_{Q}$ is the primary sex ratio in proportion females and $1-\left(\lambda+B_{Q}\right)$ is the primary sex ratio in proportion males.

When workers increase female production.-We assume that workers increase the proportion of females produced by redirecting a proportion $B_{W}$ of surplus eggs in the colony to the production of sexual females. The number of male eggs raised remains unaltered. The secondary sex ratio in colony $i$ ( $f_{i}$; eq. 1$)$ after worker biasing is

$$
f_{i}=\frac{\left(\lambda+B_{Q_{i}}\right)+B_{W_{i}}\left(\lambda+B_{Q_{i}}\right)(E-1)}{\left(\lambda+B_{Q_{i}}\right)+B_{W_{i}}\left(\lambda+B_{Q_{i}}\right)(E-1)+\left[1-\left(\lambda+B_{Q_{i}}\right)\right]}
$$

where $\left(\lambda+B_{Q}\right)$ is the proportion of eggs available that would be reared as females in the absence of worker biasing, $E$ is the ratio of eggs available to eggs reared, $\left((E-1)\left(\lambda+B_{Q}\right)\right)$ is the proportion of additional female eggs that workers could rear as females, and $\left(1-\left(\lambda+B_{Q}\right)\right)$ is the proportion of males that are reared in the absence of worker bias.

\section{Queen and Worker Biasing Costs}

\section{Queen costs}

Sex-ratio biasing is assumed to incur costs in terms of colony productivity (Ratnieks and Reeve 1992; Chapuisat et al. 1997; Reuter et al. 2004). Queen costs may arise from decreased colony growth and productivity, and increased colony mortality stemming from a limitation of female eggs available for worker production. Queen costs may also arise from the developmental costs of queen biasing mechanisms. Queen costs are assumed to increase linearly with the degree of queen bias, with a slope equal to a cost parameter $C_{Q}$.
Cost to the colony in productivity (fitness) resulting from queen bias $\left(\gamma_{Q}\right)$ is thus $\gamma_{Q}=C_{Q}\left|B_{Q}\right|$.

\section{Worker costs}

Worker costs $\left(\gamma_{W}\right)$ arise from the nonrecoverable resources invested in males prior to their elimination, or the cost of reproductive brood size reduction that can arise if workers eliminate males when resources are available to rear them in addition to any available females. They can also arise from decreased productivity due to channeling diploid eggs into the production of females rather than workers (Pamilo 1991; Reuter and Keller 2001), and from the developmental costs of biasing mechanisms. Depending upon how workers bias, their cost is either a function of the total number of available males they eliminate; or a function of the total available diploid eggs biased toward the preferred female reproductive caste.

When workers eliminate males.-The elimination of males can cause two kinds of costs. The first $\left(\gamma_{W 1}\right)$ is a mechanistic cost resulting from the resources lost by replacing male brood by female brood. This cost is assumed to increase linearly with the proportion of males eliminated. The slope of increase is $C_{W}$ and hence $\gamma_{W 1 i}=C_{W} B_{W i}\left(1-\left(\lambda+B_{Q i}\right)\right)$.

A second cost, $\gamma_{W 2}$, is due to brood reduction and arises if eliminated males cannot be replaced with females due to a lack of diploid brood. This can occur because queen biasing of the primary sex ratio (increasing male eggs) must, at some point, result in female eggs being limited (Herbers et al. 2001; Reuter and Keller 2001). The point at which male elimination results in reducing reproductive output, $B_{\text {Limit }}$, occurs where the number of eliminated males is equal to the number of surplus brood available for raising (Fig. 1). Therefore

$$
B_{\text {Limit }}=\frac{E-1}{\left[1-\left(\lambda+B_{Q}\right)\right] E} .
$$

If workers eliminate males at a proportion below $B_{\text {Limit }}$, colony productivity is reduced due to the mechanical cost $\gamma_{W 1}$ alone (i.e., $\gamma_{W i}=\gamma_{W 1 i}$ ). However, if worker biasing goes beyond that point (i.e., $B_{W}>B_{\text {Limit }}$ ), then the cost attributed to workers in colony $i, \gamma_{W i}$, is $\gamma_{W 2 i}$, where

$$
\gamma_{W 2 i}=\gamma_{W 1 i}+\left\{\left[1-\left(\gamma_{W 1 i}\right)\right]\left[1-\left(\lambda+B_{Q_{i}}\right)\right] \frac{B_{W_{i}}-B_{\text {Limit }}}{1-B_{\text {Limit }}}\right\}
$$

This equation results in a cost of $\gamma_{W 1 i}$ when $B_{W}=B_{\text {Limit }}$, that then increases in a linear manner to $1.0(100 \%$ cost and zero fitness) when $B_{Q}=-0.5$ and $B_{W}=1.0$ (when queens produce only male eggs and workers eliminate all males). An example of how costs change when workers eliminate males is illustrated in Figure 2.

When workers increase female production.-The cost due to a reallocation of worker-destined brood to female development is assumed to be a linear function of the degree of bias $\left(B_{W i}\right)$. The slope of increase is $C_{W}$, and cost is given by $\gamma_{W}=C_{W} B_{W i}\left(\lambda+B_{Q i}\right)$.

Because workers do not eliminate brood, the cost of reallocating females is not affected by brood limitation.

In our analyses, we explored the effect of costs of sex- 


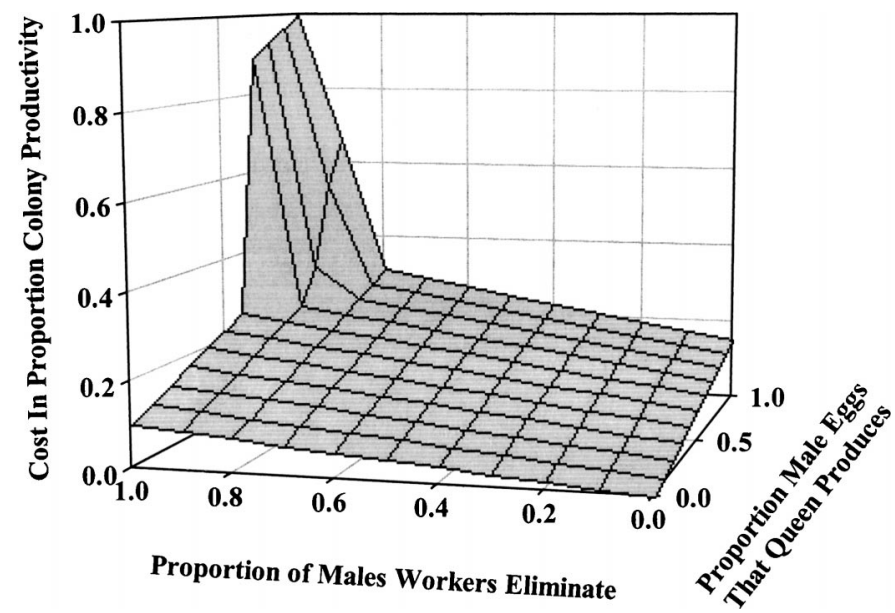

FIG. 2. Increase in the costs of sex-ratio biasing when workers eliminate males. Here, the cost parameter for queen biasing $\left(C_{Q}\right)$ is 0.3 and the cost parameter for worker biasing $\left(C_{W}\right)$ is 0.1 . There are five times more eggs available than can be reared as reproductives $(E=5)$. Costs are a linear function of the amount of bias; however, from the point where biasing by queens and workers results in reared eggs being equal to available eggs, costs rise sharply and are equal to 1.0 when queens produce only male eggs and workers eliminate all males (eq. 5).

ratio manipulation by using cost parameters, ranging from small to large, that is, from 0.1 to 0.5 , by increments of 0.1 . Because both queen and worker biasing costs affect colony productivity, colony costs ( $\gamma_{Q W}$ in eq. 1$)$ are the sum of queen and worker costs within a colony: $\gamma_{Q W}=\gamma_{Q}+\gamma_{W}$.

\section{Determining Equilibrium Bias and Sex Allocation}

We employed simulations of evolution over a large number of generations to determine equilibrium strategies. Queen and worker biasing strategies were paired randomly to form colony phenotypes, each of which produced a sex ratio defined by equations (2) or (3). The fitness of each queen and worker strategy in each colony was calculated based on the sex ratio of colony phenotypes and the population sex ratio using equation (1). The frequencies of the strategies in the next generation then either increased or decreased according to their fitness in the current generation. We assumed an arbitrarily large population size of 1,000,000 colonies. Accordingly, a strategy was eliminated (considered extinct) whenever its frequency dropped below 0.000001. Simulations were run until the frequency of biasing strategies stabilized. The number of generations required to attain equilibrium differed according to the parameters being analyzed, but generally ranged from 10,000 to 100,000 . The simulations were conducted with a computer algorithm written in Maple 6 (Waterloo Maple, Inc., Waterloo, Ontario). A more detailed explanation of the simulation procedure is supplied in the Appendix.

Our simulations started with a first generation where neither queens nor workers biased $\left(B_{W}\right.$ and $\left.B_{Q}=0\right)$. In each subsequent generation, one queen and one worker mutant arose from one randomly drawn existing (parental) biasing strategy, biasing one increment more or less than the parental strategy. Each mutation increased or decreased biasing with equal probability. Because preliminary data showed that the magnitude of incremental change in queen versus worker mutant strategies from the parental strategy affected evolutionary outcomes, we varied those increments such that they were: 0.05: 0.1 (queen $<$ worker), 0.05: 0.05 (queen $=$ worker), and 0.05: 0.025 (queen $>$ worker). Which existing strategy gave rise to a mutant strategy was determined at random, with the probability proportional to their frequency. With an assumed population size of 1,000,000 the frequency of each new mutant was 0.000001 , and this value was subtracted from the frequency of the parental strategy. Population size is important in our simulations only because it affects the probability of strategy extinction: increasing population size decreases the probability that a rare strategy is eliminated, whereas decreasing population size increases the probability that a rare strategy is eliminated. However, because eliminated strategies can arise again from mutation, population size should have little effect on the equilibrium results, except perhaps if population size were very small.

\section{RESUlts}

\section{When Workers Eliminate Males}

When workers bias the sex ratio by eliminating males, queen-worker coevolution leads to equilibrium population investment in females intermediate between the equilibria generally predicted for queens (0.5) and workers (0.75) (Fig. $3 \mathrm{~A}$ ). Surprisingly, equilibrium values are largely determined by the degree that mutations affected queen and worker biasing strategies. Population sex allocation is substantially in favor of females when mutations have a larger effect on worker biasing than queen biasing (0.05: 0.1), intermediate between the queen and worker equilibria when mutations have an equal effect on queens and worker biasing (0.05: 0.05), and close to equal investment in the sexes when mutations have a larger effect on queen biasing than on worker biasing (0.05: 0.025) (Fig. 3A).

The degree that mutations affect biasing also determines patterns of variation in individual queen and worker strategies. When mutations have a larger effect on workers than queens, queens evolve a single biasing strategy, while workers evolve two divergent strategies, with workers that do not bias and workers that generally eliminate all males (Fig. 4A). These alternative worker strategies result in split colony sex ratios, with some colonies producing both sexes and others producing all-female broods (Fig. 5A).

The sex ratio in colonies producing a mixed sex brood depends on how much queens bias the primary sex ratio in favor of males. Queens bias more at lower costs than at higher costs, and colonies produce a larger proportion of males when queen costs are low than when queen costs are high (Fig. 5A). However, because one worker strategy eliminates all males, the amount that queens can bias is also constrained by brood reduction. Queens can only bias adaptively up to a point $B_{Q}=-0.3$ because in the presence of workers that eliminate all males, any further queen bias would result in the high cost associated with brood reduction (Fig. 1). The one exception to the evolution of a single queen biasing and alternative worker biasing strategies occurs when queen costs are very high $\left(C_{Q}=0.5\right)$, in which case queens, and not workers, evolve alternative biasing strategies (Fig. 5A). The 

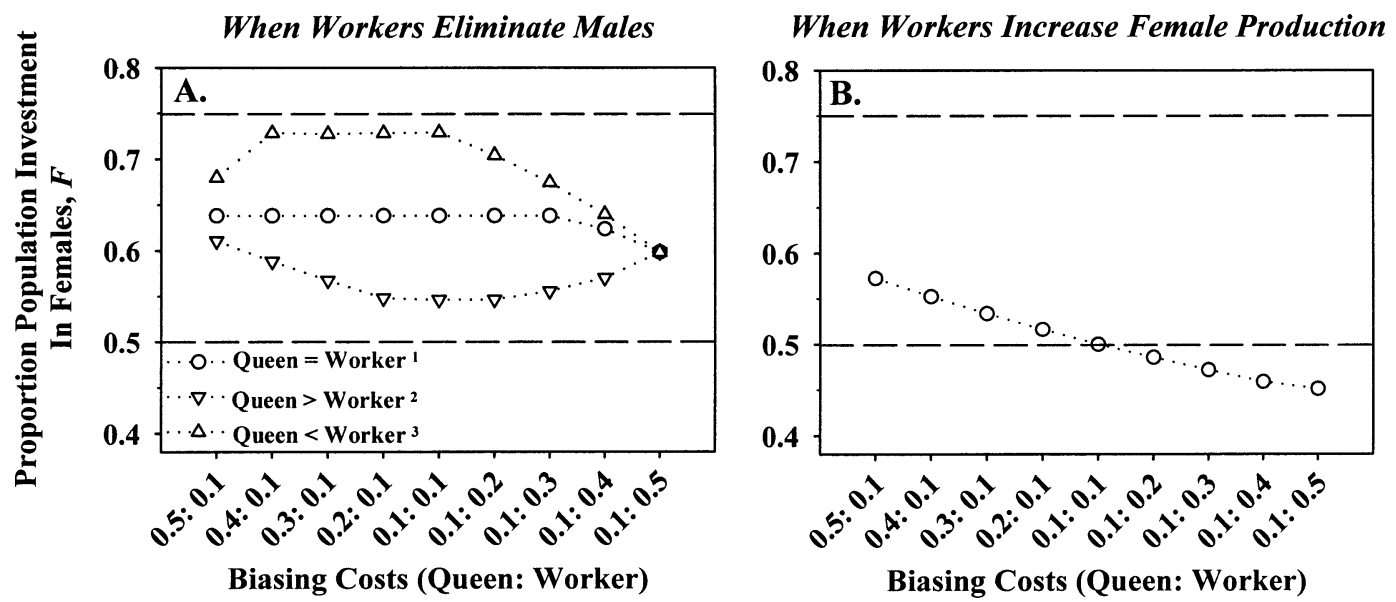

FIG. 3. Equilibrium population investment in the sexes from simulations using different queen and worker biasing costs $\left(Q_{C}\right.$, $\left.W_{C}\right)$. Dashed lines indicate the theoretical equilibrium for queens (0.5) and workers (0.75) predicted from earlier models that assume biasing has no costs (e.g., Trivers and Hare 1976).

${ }^{1}$ Incremental changes in the amount of bias are assumed equal for queen and worker mutant strategies (0.05: 0.05, queen: worker).

${ }^{2}$ Incremental changes in the amount of bias by queen mutant strategies are assumed to be greater than those of workers $(0.05$ : 0.025 , queen: worker).

3Incremental changes in the amount of bias by queen mutant strategies are assumed to be less than those of workers (0.05: 0.1, queen: worker).

degree of worker bias is equally affected by costs of manipulation, with increasing worker costs resulting in reduction of the frequency of the worker strategy eliminating all males, and decreased population level female-bias (Fig. 3A).

Alternative biasing strategies and split colony sex ratios also evolve when mutations have a larger effect on queens than workers (Figs. 4B, 5B). In these cases, two alternative queen strategies evolve, one that does not bias and another that generally produces only male eggs (Fig. 4B). The equilibrium worker response is a single biasing strategy, consisting of eliminating as many males as possible without reducing the reproductive brood $\left(B_{W}=0.8\right.$; Figs. $\left.1,4 \mathrm{~B}\right)$. As a result of queen and worker biasing, some colonies produce only males and others produce female-biased broods (Fig. $5 \mathrm{~B})$. At the population level, increasing queen biasing costs results in a decreasing frequency of queens producing only male eggs and increased investment in females (Fig. 3A).

Although monomorphic worker biasing and strong divergence in queen biasing strategies are the general rule when mutations have a larger effect on queens than workers, there are two exceptions. First, workers bias strongly at equilibrium $\left(B_{W}=0.875\right)$ when costs are low for both queens and workers $\left(C_{Q}=C_{W}=0.1\right)$, whereas the queen strategy that biases does so at a reduced level $\left(B_{Q}=-0.4\right)$, resulting in only a moderate split in colony sex ratios (Fig. 5B). The degree of queen bias is limited by the costs associated with lower production of reproductive brood (Fig 1A). Second, when worker costs are high relative to queen costs $\left(C_{W}=0.4\right)$, both queens and workers evolve alternative biasing strategies. One queen strategy produces only male eggs while the other does not bias, and one worker strategy eliminates most males while the other does not bias. Because both queens and workers evolve a nonbiasing strategy, four colony phenotypes occur, one producing mostly females, two producing only males, and one producing both sexes at equal frequency (Fig. 5B). When worker cost increases even more $\left(C_{W}=0.5\right)$, only workers evolve alternative biasing strategies. Here, one worker strategy generally eliminates all male eggs and the other does not bias, whereas queens evolve a single biasing strategy, resulting in all female- and male-biased broods (Fig. 5B).

When mutations have an equal effect on queen and worker biasing, neither queens nor workers evolve alternative biasing strategies and colony investment is unimodal unless worker costs are substantially greater than queen costs (Figs. 4C, $5 \mathrm{C})$. Over the range of costs in which unimodal investment occurs, population investment remains stable; both workers and queens bias as much as possible without reducing the reproductive brood $\left(B_{Q}=-0.35, B_{W}=0.9\right.$; Figs. 1, 3A). Increasing worker costs relative to queen costs results in split sex ratios either because of alternative queen strategies (for $C_{W}=0.4$ ), or because of alternative worker strategies (for $C_{W}=0.5$; Fig. 5C).

\section{When Workers Increase Female Production}

When workers increase the production of females, they always bias maximally at equilibrium $\left(B_{W}=1\right)$, regardless of costs. The equilibrium queen response depends on their cost of biasing relative to that of workers. When queen costs exceed worker costs, queens evolve alternative strategies with some queens producing only male eggs and the others not biasing. In this case, some colonies produce all males and others a female-biased sex ratio (Fig. 6). The proportion of queens biasing to an all-male primary sex ratio (and hence the proportion of colonies producing only males) decreases with increasing cost of queen biasing (Fig. 6), explaining why investment in females increases in the population (Fig. 3B). When queen costs are less than or equal to worker costs, queens do not exhibit alternative biasing strategies at equilibrium. Colony sex ratios are always unimodal and male biased (Figs. 3B, 5). Varying the magnitude of mutant effects on queen versus worker biasing strategies has no effect on equilibrium outcomes. 


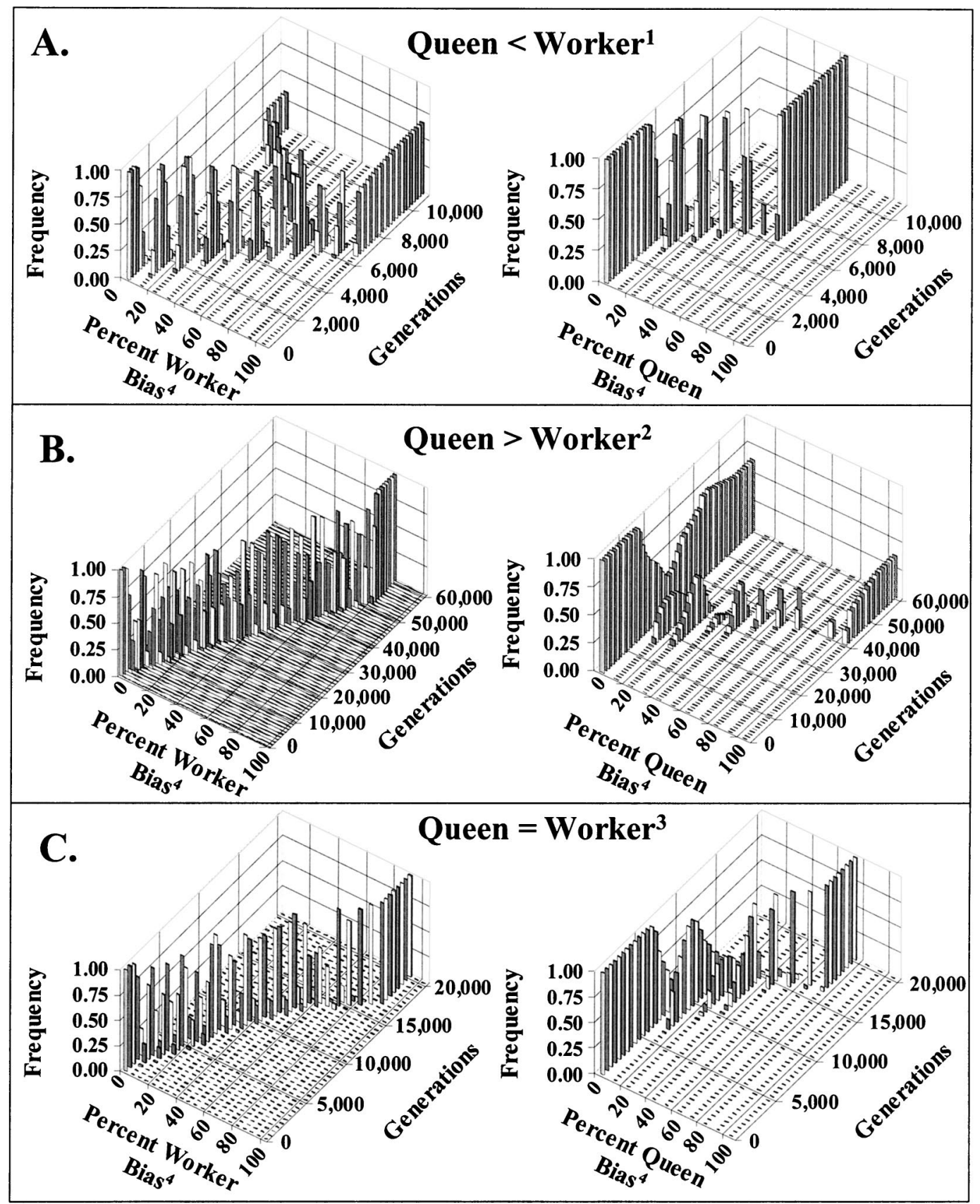

FIG. 4. Representative simulation results of the coevolution of sex-ratio biasing by queens and workers over time. In these results, workers bias by eliminating males. The cost parameter for queens $\left(C_{Q}\right)$ is 0.1 while the cost parameter for workers $\left(C_{W}\right)$ is 0.3 . Results are shown for (A) every 200 generations, (B) 1000 generations, or (C) 500 generations. Bars are alternately gray and white to aid in distinguishing between adjacent bars.

${ }^{1}$ Incremental changes in the amount of bias by queen mutant strategies are assumed to be less than those of workers $(0.05: 0.1$, queen: worker).

${ }^{2}$ Incremental changes in the amount of bias by queen mutant strategies are assumed to be greater than those of workers $(0.05: 0.025$, queen: worker).

${ }^{3}$ Incremental changes in the amount of bias are assumed equal for queen and worker mutant strategies (0.05:0.05, queen: worker).

${ }^{4}$ When the percent queen bias is zero, queens do not bias and produce an equal frequency of male and female eggs $\left(B_{Q}=0\right)$. Increasing queen bias results in increasing the proportion of male eggs, and when percent queen bias is $100\left(B_{Q}=-0.5\right)$, they produce only male eggs. When the percent worker bias is zero $\left(B_{W}=0\right)$, workers do not bias. Increasing worker bias results in increasing elimination of males, and when percent worker bias is 100 , they eliminate all males $\left(B_{W}=1.0\right)$. 
A. Larger Mutant Worker Effects ${ }^{1}$

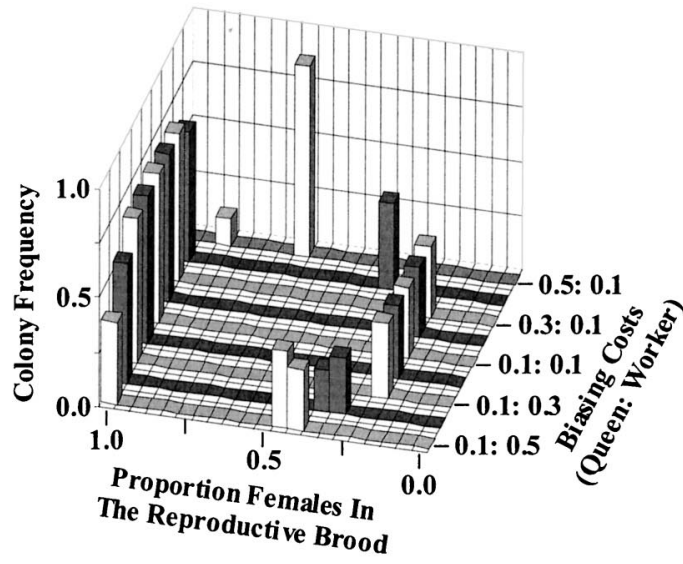

B. Larger Mutant Queen Effects ${ }^{2}$

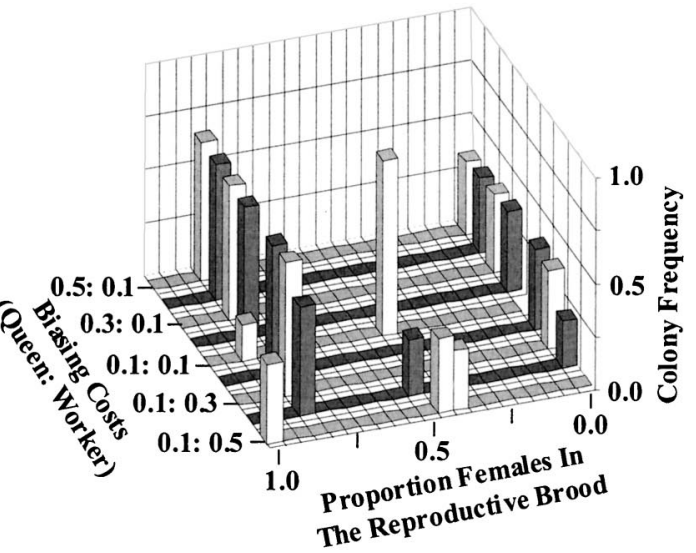

C. Equal Mutant Queen \& Worker Effects ${ }^{3}$

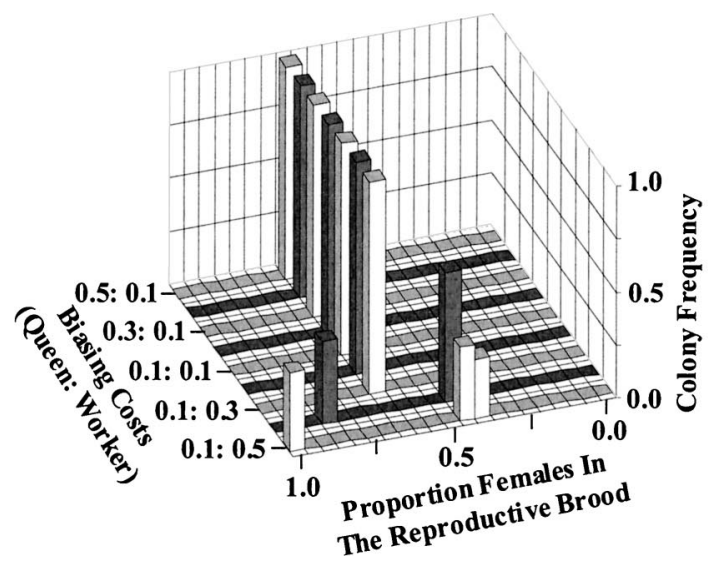

FIG. 5. Equilibrium colony investment in the sexes from simulations using different queen and worker biasing costs $\left(Q_{C}\right.$, $\left.W_{C}\right)$ when workers bias by eliminating males. Bars are alternately gray and white to aid in distinguishing between adjacent bars.

${ }^{1}$ Incremental changes in the amount of bias by queen mutant strategies are assumed to be less than those of workers (0.05:0.1, queen: worker).

${ }^{2}$ Incremental changes in the amount of bias by queen mutant strategies are assumed to be greater than those of workers (0.05:0.025, queen: worker).

${ }^{3}$ Incremental changes in the amount of bias are assumed equal for queen and worker mutant strategies (0.05:0.05, queen: worker).

\section{DISCUSSION}

\section{The Evolution of Alternative Biasing Strategies}

In our study, we modeled the resolution of queen-worker conflict over sex allocation. We asked how colony and population sex ratios evolve in response to three factors: the mechanism of sex-ratio biasing, the varying costs that queen and worker biasing incur, and the quantitative effect of mutant strategies. In evolutionary simulations, we find that split sex ratios occur frequently. Importantly, they are not inherent in our simulation procedure, because simulations with no costs consistently lead to monomorphic strategies (data not shown). Rather, the occurrence of split sex ratios depends qualitatively and quantitatively upon the evolutionary trajectory of queen and worker biasing, which in turn is dictated by the costs and benefits of biasing and the degree to which mutations increase queen and worker biasing.
In our simulations, investment in the sexes is initially equal and directional selection acts on workers to increase investment in females. This initiates a coevolutionary tug-of-war over sex allocation in which queens attempt to decrease female production while workers attempt to increase it (Fig. $4)$. If workers bias by eliminating males and the rate of evolutionary change is the same for queen and workers, this often results in a stalemate in which neither achieves their equilibrium investment and unimodal colony sex ratios occur. However, if one caste can evolve more rapidly and achieve its population-wide equilibrium, then strategies that deviate from the equilibrium on both sides (more male investment and more female investment) are favored, initiating strategy divergence and split colony sex ratios. Strategy divergence also occurs in queens when workers bias by directly increasing female caste determination.

Disruptive selection and strategy divergence occurs when 


\section{When Workers Increase Female Production}

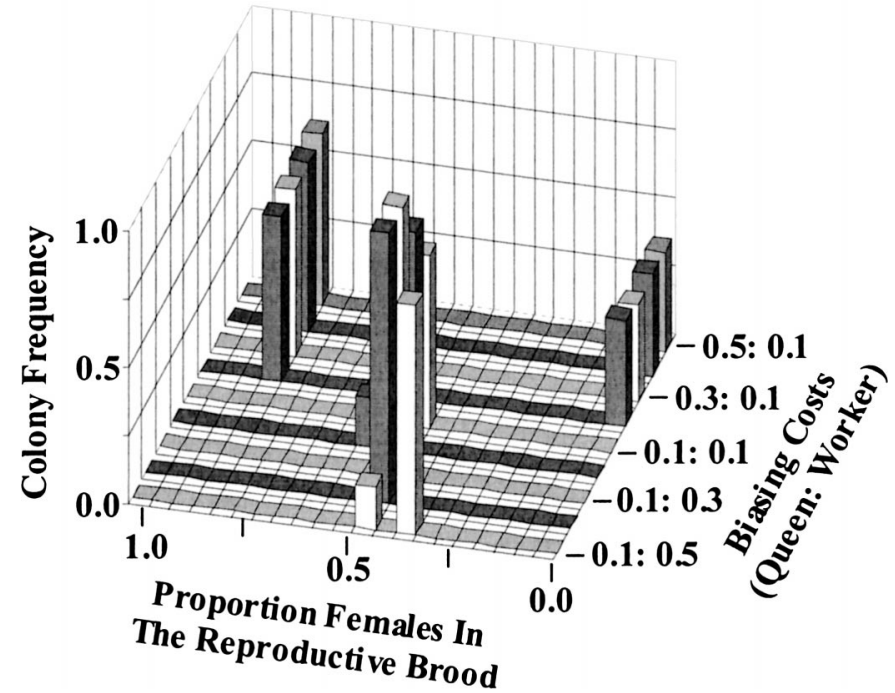

FIG. 6. Equilibrium colony investment in the sexes from simulations using different queen and worker biasing costs $\left(Q_{C}, W_{C}\right)$ when workers bias by increasing female production. Bars are alternately gray and white to aid in distinguishing between adjacent bars.

the benefit of biasing for queens (increased male production opposing worker bias) or workers (increased female production opposing queen bias) accelerates with increasing bias. For workers this occurs when they eliminate males and for queens it occurs under both worker-biasing mechanisms (Figs. 7A,B,D). Because the cost of biasing increases in a linear manner, accelerating benefits result in biasing becoming increasingly cost-effective with increasing bias, meaning that the more one biases, the less it costs per unit effect. Increasing cost effectiveness causes disruptive selection in biasing strategies once the population has reached the population-level sex ratio equilibrium. At this point, strategies deviating from the population-level equilibrium have greater fitness than a strategy matching it. Strategies biasing beyond the equilibrium gain because they benefit from increasing cost efficiency and a greater proportion of the preferred sex. In contrast, strategies biasing less than the equilibrium have greater fitness because they have smaller overall biasing costs and benefit from greater colony productivity. Despite the divergence of individual strategies, a population-level equilibrium in sex allocation is maintained by selection on the frequency of the divergent alternative strategies (also see Reuter et al. 2004).

When workers bias by eliminating males, both queens and workers exhibit accelerating benefits and yet only one caste generally evolves alternative strategies. This occurs because the caste that first reaches an equilibrium evolves alternative strategies first, and then the other caste cannot bias strongly enough to reach their equilibrium without causing brood reduction (i.e., where $B_{W}>B_{\text {Limit }}$ ). For example, one of two alternative queen strategies is the production of an all-male sex ratio, which constrains workers to levels of male elim- ination that do not result in brood reduction in all-male colonies. Similarly, one of two alternative worker strategies is to eliminate all males. This strategy prevents queens from evolving strongly male-biased egg sex ratios because this would also result in brood reduction.

The reason that neither caste generally evolves alternative strategies when mutations have an equal effect on queen and worker biasing is that population equilibria for each caste increase at approximately the same rate as biasing by the opposing caste. At any point where biasing by one caste exceeds their equilibrium (initiating strategy divergence), the other caste also biases equally strongly, resetting the degree of bias necessary to reach equilibrium to a higher value. Accordingly, divergence is no longer favored and directional selection again acts to increase bias towards the new equilibrium, with the effect that each caste prevents the other from evolving strategy divergence. The end result is that neither caste evolves alternative strategies and at equilibrium both queens and workers bias as much as possible without reducing the reproductive brood.

Which caste reaches their equilibrium first, and thus which caste evolves alternative strategies, depends partly on the rate at which strategies can evolve, with the caste exhibiting larger mutational changes in biasing reaching equilibrium more quickly. Accordingly, alternative queen strategies tend to evolve when the effects of mutations are greater in queens than workers, and workers evolve alternative strategies in the opposite case (Figs. 4A,B, 5, and 6). However, the cost of biasing is another determinant of how quickly each caste reaches equilibrium. With large costs, changes in colony investment generate costs that outweigh the benefit of biasing (Reuter et al. 2004), resulting in a population equilibrium occurring at low bias, and early initiation of strategy divergence. This is evidenced by our finding that extreme differences in costs between castes can reverse the effect of differences in the degree to which mutation affects bias. Larger mutational steps in workers than queens, for example, usually lead to divergent worker strategies. However, strategy divergence is observed in queen and not worker strategies when biasing is much more costly for queens than workers, and in worker but not queen strategies when biasing is much more costly for workers than queens.

When workers bias by increasing female production, the costs of biasing are also important in explaining why queens do not evolve alternative biasing strategies when their biasing costs are less than those of workers (Fig. 6). The reason is that increasing queen bias decreases the availability of diploid eggs, which decreases the degree that workers can bias. Hence, increased queen bias at low cost prevents workers from biasing at higher cost, and colony productivity increases with increasing queen bias. Under this condition, a queen strategy that biases beyond a population equilibrium will still bias more cost-effectively than biasing at equilibrium; however, a strategy biasing below the equilibrium will not have lower costs, and this prevents the evolution of alternative strategies.

The cost-saving effect of increasing queen bias is also the cause of male-biased population-level investment when workers bias by increasing female production (Fig. 3B). When worker costs are greater than queen costs, queens' bias 


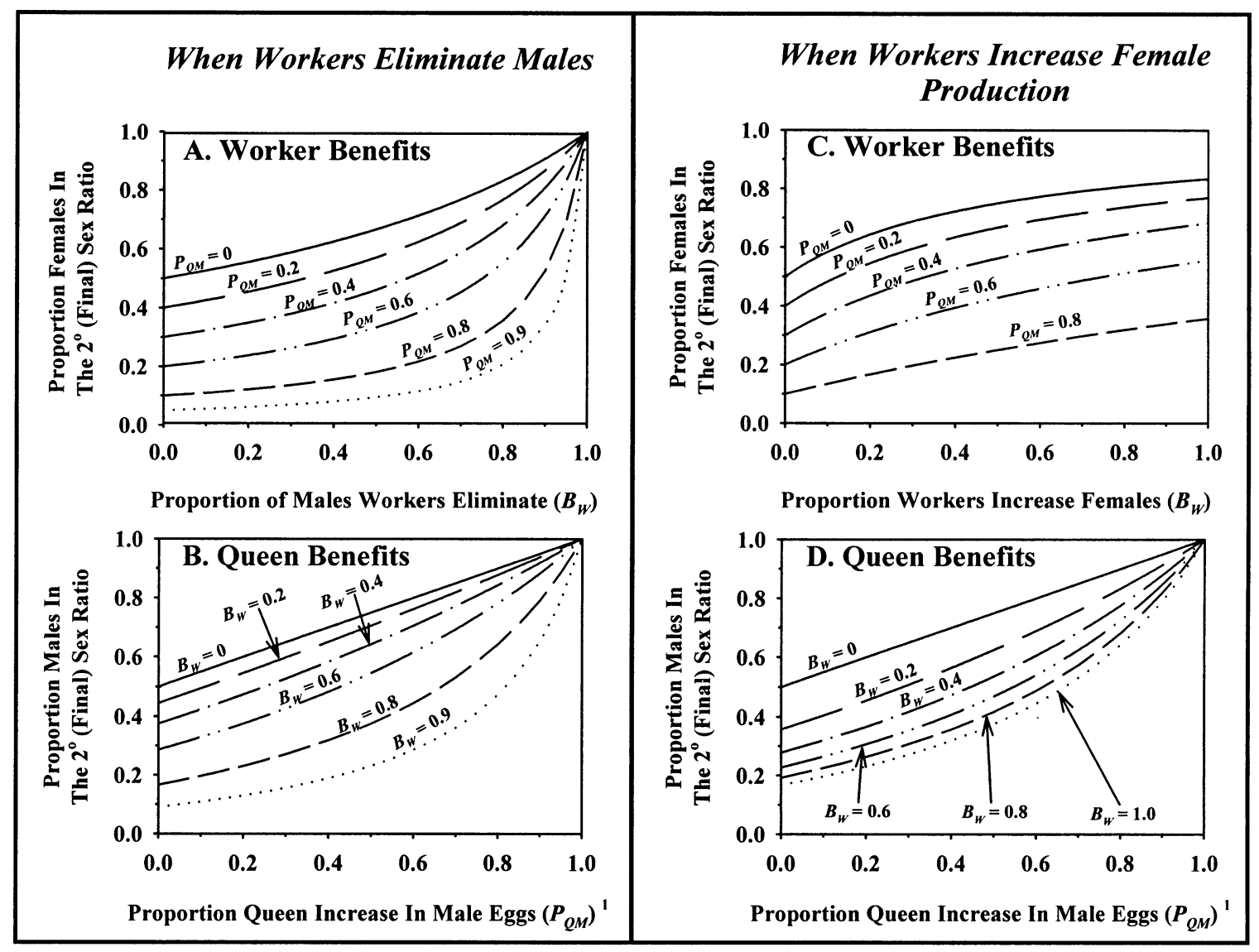

FIG. 7. Changes in final colony investment in the sexes according to how much queens and worker bias. (A) and (C) According to worker bias at various degrees of queen bias. (B) and (D) According to queen bias at various degrees of worker bias. $P_{Q M}$ is the proportion queen bias, where $P_{Q M}=0.0$ corresponds to a lack of queen biasing and a primary (egg) sex ratio of equality (i.e., $\lambda$ ), and $P_{Q M}=1.0$ corresponds to maximal queen bias and the production of only male eggs. $B_{W}$ is the proportion of males that workers eliminate. Secondary (final) sex ratios are derived from equations (2) and (3).

beyond what would result in equal investment in the sexes because the sum of queen and worker costs are less than when biasing to the point of equal investment. This result may seem odd, because the fitness of both queens and workers would be greater if neither biased at all. However, it occurs because a mutant queen strategy producing fewer male eggs (resulting in more equal population investment) would increase the amount workers can effectively bias, which would again select for increased queen bias.

Given the importance that the effects of mutations on biasing have for the resolution of sex-ratio conflict, the question arises whether in nature queens and workers ever differ in this regard. In the absence of any knowledge of the genetics of queen and worker behavior, it is obviously impossible to make any definite statement on this point. However, assuming that queens and workers utilize substantially different mechanisms to bias colony investment, the genetic basis for these traits and the numbers of genes involved in their expression are likely to differ. If so, those differences could frequently result in differences in the degree that mutations affect biasing phenotypes, and thus in differences in the rate at which queen and worker biasing strategies can evolve. Nevertheless, it is important to note that the quantitative effects of mutations on queen and worker biasing strategies could vary over time rather than exhibiting the consistency we model. If mutations vary such that they sometimes have a greater effect on queen biasing while sometimes having a greater effect on worker biasing, the overall rate of evolutionary change resulting from the effects of mutations on biasing will become more equal for queens and workers, which should decrease the probability of disruptive selection and the evolution of alternative biasing strategies.

Central to our study is the assumption that eggs are limited but that queens must produce them in excess of those that can be reared as reproductives. In our simulations, we assumed that eggs were five times more frequent than those that can be reared as reproductives. What happens when eggs are more or less frequent? When workers eliminate males, lower egg availability reduces the probability that queens or workers can bias to the degree necessary to reach a population equilibrium prior to the point where the cost of brood reduction prohibits further biasing (Figs. 1,2). Lower egg availability thus reduces the probability of the evolution of alternative biasing strategies and split colony sex ratios (Fig. 1). Conversely, increased egg availability increases that probability. However, both cases also depend upon the relative effect of queen versus worker mutations. At any given ratio of available to reared eggs greater than one, larger differences 
in the effects of mutations increases the probability of the evolution of split colony sex ratios, while a lesser difference decreases that probability.

It is important to note that Roisin and Aron (2003) have also recently modeled a case in which queens bias the primary sex ratio and workers eliminate males to bias the secondary sex ratio. Their study also predicted the evolution of alternative queen (but not worker) biasing strategies. However, their analytical approach and conclusions differ greatly from ours. Most important, they did not consider worker biasing to have costs, and costs were only attributed to queens and occurred only if they produced fewer diploid eggs than necessary to optimize worker production (Roisin and Aron 2003).

\section{Study Predictions}

Our study shows that the evolution of queen-worker conflict can involve considerable complexity. However, there are at least three clearly testable predictions. First, our study predicts that the nature of split colony sex ratios at or near equilibrium should be diagnostic of whether queens or workers exhibit alternative biasing strategies. Strongly male-biased broods co-occurring with more mixed-sex broods should indicate that queens exhibit alternative biasing strategies. Conversely, strongly female-biased broods co-occurring with more mixed-sex broods should indicate that workers exhibit alternative biasing strategies. There is a clear need for experiments to test for the presence of alternative biasing strategies and to determine whether queens or workers exhibit them. There is, however, evidence that they exist, and there are procedures for discovering them (Helms et al. 2000; Passera et al. 2001).

Second, our study predicts that colonies producing an extreme sex-ratio bias will be less productive than colonies producing a greater mix of the two sexes. When queens exhibit alternative biasing strategies and workers do not, a colony phenotype with strongly male-biased investment results from biasing queens co-occurring with biasing workers, and the cost in colony productivity is the sum of both queen and worker costs. Because only workers bias in colonies producing more mixed-sex broods, colony productivity will be greater. Just the opposite should occur when workers exhibit alternative biasing strategies. In this case, colonies producing strongly female-biased sex ratios will contain queens and workers that bias, and will thus be less productive than colonies with more mixed-sex broods, where only queens bias.

Third, when workers bias by eliminating males, our study also predicts an association between whether queens or workers exhibit alternative biasing strategies and population sex allocation. The evolution of alternative biasing strategies in queens reduces the ability of workers to effectively influence the sex ratio, resulting in population level sex allocation closer to equality than to 0.75 (Fig. 3A). In contrast, the evolution of alternative biasing strategies in workers reduces the ability of queens to effectively influence the sex ratio, resulting in population level sex allocation closer to 0.75 than to equality (Fig. 3A). Whether this is the case could be tested with individual populations or, because strong estimates of population investment are often difficult for eusocial Hymenoptera
(Boomsma 1989, Helms 1994), with comparative analyses among populations or species where either queens or workers exhibit alternative biasing strategies.

It is important to note that not all of these predictions are unique to our models. For example, strongly male-biased broods co-occurring with more mixed-sex broods may also be predicted under sex ratio compensation when some colonies lose their queen (Taylor 1981). Queen loss could also result in lower productivity in colonies producing extremely male-biased broods. However, sex ratio compensation uniquely predicts that male producing colonies will lack a functional queen. Split colony sex ratios may also occur because workers bias the colony sex ratio according to variable relatedness asymmetry (Boomsma and Grafen 1991). Given various conditions, this model can predict a variety of colony sex ratios consistent with our results. However, the variable relatedness asymmetry model uniquely predicts that relatedness asymmetry will be greater in colonies producing primarily or exclusively females than in those producing primarily or exclusively males' (Boomsma and Grafen 1991). It also fails to predict the evolution of alternative queen biasing strategies or an association between colony sex ratio and colony productivity in the direction predicted by our results.

Split sex ratios have also been hypothesized to result from local mate competition (LMC) or local resource competition (LRC), where the rate of return on investment in the sexes differs according to overall colony investment (Frank 1987). Under LMC, small colonies are predicted to produce only males and larger colonies both sexes; and colonies producing extremely male-biased sex ratios should be less productive than colonies producing a greater mix of the two sexes (Frank 1987). Under LRC, small colonies are predicted to produce only females and larger colonies a mix of the two sexes; and colonies producing extremely female-biased sex ratios should be less productive than colonies producing a greater mix of the two sexes (Frank 1987). These are also predictions of our model. However, the LMC and LRC models do not predict any clear association between whether queens or workers exhibit alternative biasing strategies and colony sex ratio. In addition, our prediction that queens will exhibit alternative biasing strategies in populations in which population investment in the sexes is near equal, while workers will exhibit alternative biasing strategies in populations in which investment is substantially female-biased, appears unique in existing theory.

\section{Alternative Mechanisms and Social Structures}

Our study focuses on biasing the primary sex ratio as the mechanism of queen bias. However, in small colony Hymenoptera other mechanisms, including direct queen policing of worker behavior, might be possible and how our conclusions may apply to such cases is unclear. In addition, our study has focused on a simple social structure where workers are sterile and colonies have a single queen inseminated by a single male, excluding any variation in relatedness. What our models might predict for eusocial Hymenoptera with alternative social structures remains to be determined. When multiple mating by queens or multiple reproductive queens 
per colony are frequent, alternative queen and/or worker strategies might often occur within single colonies, and outcomes may depend upon factors such as the relatedness and degree of reproductive skew among queens and behavioral dominance among worker phenotypes. In addition, variable relatedness asymmetry within populations can result in the evolution of split colony sex ratios (Boomsma and Grafen 1991), and how the mechanisms we address might influence that process remains to be explored. However, our approach of analyzing queen-worker conflict from a mechanistic perspective provides a foundation for addressing such questions and for studying of the evolution of sex allocation over a variety of different social structures.

\section{ACKNOWLEDGMENTS}

We thank S. Helms Cahan for many helpful discussions, and J. J. Boomsma, M. Chapuisat, R. L. Hammond, S. Helms Cahan, P. Nonacs, U. Mueller, and anonymous reviewers for very constructive comments on the manuscript. The research was supported by the Swiss National Science Foundation, and M. Reuter acknowledges support from the European Commission in the form of a Marie Curie fellowship.

\section{Literature Cited}

Aron, S., L. Passera, and L. Keller. 1994. Queen-worker conflict over sex ratio: a comparison of primary and secondary sex ratios in the Argentine ant, Iridomyrmex humilis. J. Evol. Biol. 7: 403-418.

Aron, S., E. L. Vargo, and L. Passera. 1995. Primary and secondary sex ratios in monogyne colonies of the fire ant, Solenopsis invicta. Anim. Behav. 49:749-757.

Beekman, M., and F. L. W. Ratnieks. 2003. Power over reproduction in social Hymenoptera. Phil. Trans. R. Soc. Lond. B. 358: 1741-1753.

Boomsma, J. J. 1989. Sex-investment ratios in ants: has female bias been systematically overestimated? Am. Nat. 133:517-532.

Boomsma, J. J., and A. Grafen. 1990. Intraspecific variation in ant sex ratios and the Trivers- Hare hypothesis. Evolution 44: 1026-1034.

_ 1991 . Colony-level sex ratio selection in eusocial Hymenoptera. J. Evol. Biol. 4:383-407.

Bourke, A. F. G., and N. R. Franks. 1995. Social evolution in ants. Princeton Univ. Press, Princeton, NJ.

Bourke, A. F. G., and F. L. W. Ratnieks. 2001. Kin-selected conflict in the bumble-bee Bombus terrestris (Hymenoptera: Apidae). Proc. R. Soc. Lond. B 266:347-355.

Bulmer, M. G. 1981. Worker-queen conflict in annual social Hymenoptera. J. Theoret. Biol. 93:239-251.

Chan, G. L., and A. F. G. Bourke. 1994. Split sex ratios in a multiple-queen ant population. Proc. R. Soc. Lond. B 258:261-266.

Chapuisat, M., and L. Keller. 1999. Testing kin selection with sex allocation data in eusocial Hymenoptera. Heredity 82:473-478.

Chapuisat, M., L. Sundström, and L. Keller. 1997. Sex ratio regulation: the economics of fratricide in ants. Proc. R. Soc. Lond. B 264:1255-1260.

Cheverud, J. M. 2003. Evolution in a genetically heritable social environment. Proc. Natl. Acad. Sci. 100:4357-4359.

Cremer, S., and J. Heinze. 2002. Adaptive production of fighter males: queens of the ant Cardiocondyla adjust the sex ratio under local mate competition. Proc. R. Soc. Lond. B 269:417-422.

Crozier, R. H., and P. Pamilo. 1996. Evolution of social insect colonies: sex allocation and kin selection. Oxford Univ. Press, Oxford, U.K.

Fisher, R. A. 1958. The genetical theory of natural selection, 2nd ed. Dover Press, New York.

Fournier, D., L. Keller, L. Passera, and S. Aron. 2003. Colony sex ratios vary with breeding system by not relatedness asymmetry in the facultatively polygynous ant Pheidole pallidula. Evolution 57:1336-1342.

Frank, S. A. 1987. Variable sex ratio among colonies of ants. Behav. Ecol. Sociobiol. 20:195-201.

Grafen, A. 1986. Split sex ratios and the evolutionary origins of eusociality. J. Theoret. Biol. 122:95-121.

Hamilton, W. D. 1964. The genetical evolution of social behaviour. I and II. J. Theoret. Biol. 7:1-52.

. 1967. Extraordinary sex ratios. Science 156:477-488.

Hammond, R. L., M. W. Bruford, and A. F. G. Bourke. 2002. Ant workers selfishly bias sex ratios by manipulating female development. Proc. R. Soc. Lond. B 269:173-178.

Hasegawa, E. 1994. Sex allocation in the ant Colobopsis nipponicus (Wheeler). 1. Population sex ratio. Evolution 48:1121-1129.

Helms, K. R. 1994. Sexual size dimorphism and sex ratios in bees and wasps. Am. Nat. 143:418-434.

- 1999. Colony sex ratios, conflict between queens and workers, and apparent queen control in the ant Pheidole desertorum. Evolution 53:1470-1478.

Helms, K. R., J. H. Fewell, and S. W. Rissing. 2000. Sex ratio determination by queens and workers in the ant Pheidole desertorum. Animal Behaviour 59:523-527.

Helms, K. R., D. Fournier, D., L. Keller, L. Passera, and S. Aron. 2004. Colony sex ratios in the facultatively polygynous ant Pheidole pallidula: a reanalysis with new data. Evolution 58: 1141-1142.

Herbers, J. M., C. J. DeHeer, and S. Foitzik. 2001. Conflict over sex allocation drives conflict over reproductive allocation in perennial social insect colonies. Am. Nat. 158:178-192.

Keller, L., S. Aron, and L. Passera. 1996. Inter-nest sex ratio variation and male brood survival in the ant Pheidole pallidula. Behav. Ecol. 7:292-298.

Kölliker, M., M. W. G. Brinkhof, P. Heeb, P. S. Fitze, and H. Richner. 2000. The quantitative genetic basis of offspring solicitation and parental response in a passerine bird with biparental care. Proc. R. Soc. Lond. B 267:2127-2132.

Lynch, M. 1987. Evolution of intrafamilial interactions. Proc. Natl. Acad. Sci. 84:8507-8511.

Mehdiabadi, N. J., H. K. Reeve, and U. G. Mueller. 2003. Queens versus workers: sex ratio conflict in eusocial Hymenoptera. Trends Ecol. Evol. 18:88-93.

Mock, D. W., and G. A. Parker. 1997. The evolution of sibling rivalry. Oxford Univ. Press, Oxford, New York.

Mueller, U. G. 1991. Haplodiploidy and the evolution of facultative sex ratios in a primitively eusocial bee. Science 254:442-444.

Nonacs, P. 1986. Ant reproductive strategies and sex allocation theory. Quart. Rev. Biol. 61:1-21.

Pamilo, P. 1991. Evolution of colony characteristics in social insects. I. Sex allocation. Am. Nat. 137:83-107.

Pamilo, P., and R. Rosengren. 1983. Sex ratio strategies in Formica ants. Oikos 40:24-35.

Pamilo, P., and P. Seppä. 1994. Reproductive competition and conflicts in colonies of the ant Formica sanguinea. Anim. Behav. 48:1201-1206.

Passera, L, S. Aron, E. L. Vargo, and L. Keller. 2001. Queen control of sex ratio in fire ants. Science 293:1308-1310.

Passera, L., and S. Aron. 1996. Early sex discrimination and male brood elimination by workers of the Argentine ant. Proc. R. Soc. Lond. B 263:1041-1046.

Queller, D. C., and J. E. Strassmann. 1998. Kin selection and social insects. BioScience 48:165-175.

Queller, D. C., J. E. Strassmann, C. R. Solis, C. R. Hughes, and D. M. DeLoach. 1993. A selfish strategy of social insect workers that promotes social cohesion. Nature 365:639-641.

Ratnieks, F. L. W., and H. K. Reeve. 1992. Conflict in single-queen Hymenopteran societies: the structure and processes that reduce conflict in advanced eusocial species. J. Theoret. Biol. 158: 33-65.

Reuter, M., and L. Keller. 2001. Sex Ratio conflict and worker production in eusocial Hymenoptera. Am. Nat. 158:166-177.

Reuter, M., K. R. Helms, L. Lehmann, and L. Keller. 2004. Effects of brood manipulation costs on optimal sex allocation in social Hymenoptera. Am. Nat. 164:E73-E82. 
Roisin, Y, and S. Aron. 2003. Split sex ratios in perennial social Hymenoptera: a mixed evolutionary stable strategy from the queens' perspective? Am. Nat. 162:624-637.

Sundström, L. 1994. Sex ratio bias, relatedness asymmetry and queen mating frequency in ants. Nature 367:266-268.

Sundström, L, M. Chapuisat, and L. Keller. 1996. Conditional manipulation of sex ratios by ant workers: a test of kin selection theory. Science 274:993-994.

Taylor, P. D. 1981. Sex ratio compensation in ant populations. Evolution 35:1250-1251.

Trivers, R. L., and H. Hare. 1976. Haplodiploidy and the evolution of the social insects. Science 191:249-263.

Vargo, E. L. 1996. Sex investment ratios in monogyne and polygyne populations of the fire ant Solenopsis invicta. J. Evol. Biol. 9: 783-802.

Corresponding Editor: U. Mueller

\section{APPENDIX}

Simulation procedures for the evolution of queen-worker sex allocation conflict.

Our simulation procedure first randomly pairs queen and worker strategies within colonies, and the frequency of each of these colonies is determined by multiplying the population frequency of the queen strategies by the population frequency of the worker strategies. Colony sex ratios are calculated for all colonies $(1-N)$ from either equation (2) or (3), depending upon how workers bias. The population sex ratio is then determined by multiplying the frequency $(\phi)$ of each colony phenotype by the proportion females they produce $(f)$ by the proportion of resources invested in reproduction $\left(1-\gamma_{Q W}\right)$ and summing that value across all colony phenotypes, that is, by:

$$
\sum \text { Population Investment In Females }=\sum_{\text {Colony } Q_{1} W_{1}}^{{\text {Colony } Q_{N} W_{N}}_{N}} \phi f\left(1-\gamma_{Q W}\right)
$$

Population investment in males is determined the same way, except investment in males is $1-f$ :

$\sum$ Population Investment In Males $=\sum_{\text {Colony } Q_{1} W_{1}}^{\text {Colony } Q_{N} W_{N}} \phi(1-f)\left(1-\gamma_{Q W}\right)$

The proportional investment in females within the population $(F)$ is then determined by:

$$
\begin{gathered}
F=\left(\sum \text { Population Investment In Females }\right) \\
\div\left(\sum\right. \text { Population Investment In Females } \\
\left.\quad+\sum \text { Population Investment In Males }\right)
\end{gathered}
$$

It is important to note that population investment in females $(F)$ is not simply the sum of the proportion investment in females, because different colonies can bias differently and have different costs, and as a result, investments have to weighted by colony productivity.

Once $F$ is determined, the fitness of queen and worker strategies is determined in all of the colony phenotypes. The frequency of each queen strategy in the next generation is then calculated by the sum of their frequency in the last generation multiplied by their fitness in the current generation $\left(\alpha_{Q}\right)$. For each queen strategy, this is divided by the sum of the frequency of all queen strategies in the last generation $\left(\phi_{O}\right)$ multiplied by their fitness in the current generation. The frequency of queen strategy 1 in the next generation $\left(Q_{1(\mathrm{New})}\right)$ is:

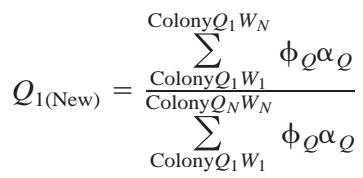

The frequency of each worker strategy in the next generation is calculated by using the same procedure as for queens. The new frequency of worker strategy 1 is:

$$
W_{1(\text { New })}=\frac{\sum_{\text {Colony } Q_{1} W_{1}}^{\text {Colony } Q_{N} W_{1}} \phi_{W} \alpha_{W}}{\sum_{\text {Colony } Q_{1} W_{1}}^{\text {Colon } W_{N}} \phi_{W} \alpha_{W}}
$$

The simulation then uses the new frequencies of queen and worker strategies to form new colony phenotypes, and the simulation procedure is repeated until the number of generations specified has been completed. The simulation then provides output on the final frequency of the different queen and worker strategies, the final frequency of the colony phenotypes, the final fitness of the queen and worker strategies, population investment $(F)$, and colony investment by each of the colony phenotypes. 